\title{
Distribution, pharmacokinetics and primary metabolism model of tramadol in zebrafish
}

\author{
HUIQIN ZHUO ${ }^{1 *}$, HONGWEI JIN ${ }^{2 *}$, HUIFANG PENG ${ }^{3}$ and HEQING HUANG ${ }^{3-5}$ \\ ${ }^{1}$ Department of Gastrointestinal Surgery, Zhongshan Hospital, Xiamen University, Xiamen, Fujian 361004; \\ ${ }^{2}$ Center of Clinical Laboratory, Zhongshan Hospital; ${ }^{3}$ State Key Laboratory of Stress Cell Biology, School of Life Science; \\ ${ }^{4}$ State Key Laboratory of Marine Environmental Science, College of Oceanography and Environmental Science; \\ ${ }^{5}$ The Key Laboratory for Chemical Biology of Fujian, College of Chemistry \& Chemical Engineering, \\ Xiamen University, Xiamen, Fujian 361005, P.R. China
}

Received October 9, 2015; Accepted October 12, 2016

DOI: $10.3892 / \mathrm{mmr} .2016 .5956$

\begin{abstract}
The current study aimed to develop a rapid, robust and adequately sensitive method for simultaneous determination of the concentration of tramadol and its active metabolites in zebrafish. The pharmacokinetic and elimination pattern of tramadol and its major phase I metabolites following oral or intramuscular administration in zebrafish tissues was achieved using electrospray ionization-quadrupole-time of flight/mass spectrometry (ESI-Q-TOF/MS) and gas chromatography/mass spectrometry (GC-MS). Following administration, the metabolisms were detected in the brain, eyes, muscle and gill tissues within $1 \mathrm{~h}$. Two tramadol metabolites, O- and $\mathrm{N}$-desmethyltramadol, were detected in brain tissue, with $\mathrm{N}$-desmethyltramadol detected at a higher level. Following GC-MS detection the curve indicated an initial rapid phase, corresponding to the detection of the tramadol within $1 \mathrm{~min}$, and reached peak value in the brain at $5 \mathrm{~min}$. Faster drug clearance was detected in low-dose groups, and concentration had dropped around the to initial level $(1.11 \mu \mathrm{g})$ at $20 \mathrm{~min}$, but was detectable for up to $3 \mathrm{~h}$. However, it took $80 \mathrm{~min}$ to fall back to the initial value $(1.73 \mu \mathrm{g})$ in the high-dose groups, and tramadol was detectable for up to $4 \mathrm{~h}$. This study developed and validated a simple and high throughput analytical procedure to determine the distribution and pharmacokinetic profiles of tramadol, and its primary metabolites in tissues of zebrafish.
\end{abstract}

Correspondence to: Dr Heqing Huang, The Key Laboratory for Chemical Biology of Fujian, College of Chemistry \& Chemical Engineering, Xiamen University, 422 Siming South Road, Xiamen, Fujian 361005, P.R. China

E-mail: huangheqing20158@sina.com

${ }^{*}$ Contributed equally

Key words: tramadol, ESI-Q-TOF/MS, zebrafish, GC-MS

\section{Introduction}

Tramadol hydrochloride (TH), an atypical, centrally acting, opioid analgesic, used in the treatment of moderate to severe acute and chronic pain, has become the most prescribed opioid worldwide $(1,2)$. It possesses a weak affinity for the $\mu$ - and $\delta$-opioid receptors, and weaker affinity for the $\kappa$-subtype. It also interferes with the neuronal release and reuptake of serotonin and norepinephrine, which appears to contribute to the analgesic effect (3). Tramadol is extensively metabolized in the liver. The primary metabolites, O-desmethyltramadol (M1) and N-desmethyltramadol (M2), may be further metabolized to three additional secondary metabolites, namely, N, N-didesmethyltramadol (M3), N,N, O-tridesmethyltramadol (M4) and N,O-didesmethyltramadol (M5). The clinical response to tramadol is closely correlated with its metabolism. M1 is reported to be the major active metabolite and it is 200 times more potent at the $\mu$-receptor than the parent drug, tramadol (4). Despite the long-term use of tramadol, and lack of characteristic opiate side effects, the knowledge and prediction of the time-course of its pharmacologic effects are hampered by the presence of active metabolites and the coexistence of opioid and nonopioid mechanisms. Furthermore, the adverse effects of tramadol have been increasingly reported, and data obtained from postmarketing surveillance, case reports and laboratory tests has indicated the abuse of tramadol has increased (5-7). These issues highlight the need of an appropriate strategy for screening the addictive potential and neurotoxicity of tramadol in animals and human.

Zebrafish (Danio rerio) have been widely used as an experimental model in molecular biology and development, and to investigate the molecular basis of certain human diseases. Zebrafish represent an established model for studying nociceptive responses, they not only have the necessary sensory components for perceiving potentially painful stimuli, but also possess the biochemical pathways important for transmitting and interpreting pain in higher vertebrates (8). As vertebrates, zebrafish are structurally homologous to humans, and their genes are $70-80 \%$ identical to human counterparts (9). It is a valid system to discover and validate novel pharmacological targets, and to identify novel drugs, perform toxicological 
tests, and to analyze the biological effects of several abused drugs, including cocaine, ethanol and morphine (10-12). Gonzalez-Nuñez et al (13) cloned and characterized four opioid receptors, and five opioid drug precursors in zebrafish, which are homologous to their mammalian counterparts. Therefore, zebrafish are likely to be an appropriate experimental model to analyze the biological effects of abuse and neurotoxicity of tramadol, however, initially the pharmacokinetic parameters of tramadol in zebrafish need to be established.

A variety of quantitative bioanalytical methods have been reported to determine tramadol concentration alone or combination with its metabolites in various tissues, such as brain and gut. Methods for estimating the tramadol concentration alone or in combination with its metabolites have been previously described, employing high performance liquid chromatography (HPLC) with UV, fluorescence and diode array detector, gas chromatography (GC) with flame ionization detection, and mass spectrometry (GC-MS) detection, liquid chromatography-mass spectrometry (LC-MS), LC-MS/MS, capillary electrophoresis, thin layer chromatography and flow injection analysis systems (14-16). The majority of these previous studies focus their attention exclusively on tramadol and its active metabolite, M1, in biological fluids, including saliva, urine, amniotic fluid and plasma, however, to the best of our knowledge, only two studies have reported the determination of tramadol and metabolite levels in the brain tissue of mice and rats by GC-nitrogen-phosphorus detection $(17,18)$.

Simultaneous quantification of tramadol and its metabolites has been performed in plasma and/or urine of humans, horses, dogs, camels, rabbits, mice, amphibians, rats, cats and donkeys (19-22). The active metabolite M1 in plasma in various animal species is low, whereas it is high in human plasma. Tramadol has been previously reported to be metabolized faster to inactive metabolites in goats, dogs and horses, than in cats. Furthermore, studies demonstrating the pharmacokinetics of tramadol in camels, horses and donkeys report high plasma levels of tramadol, but negligible plasma concentrations of the M1 metabolite, questioning the clinical effectiveness of the drug as an analgesic in these species (23). Pharmacokinetic studies indicate interspecies differences in drug metabolism, supporting the fact that pharmacokinetic studies are necessary for each species, and in a variety of different tissues. However, very few sensitive methods are currently established for the detection of tramadol and its active metabolite in zebrafish, particularly in tissues.

Thus, there is a need to develop rapid, robust and adequately sensitive methods for simultaneous determination of tramadol levels and its active metabolites in zebrafish. The current study aimed to develop rapid electrospray ionization-quadrupole-time of flight/mass spectrometry (ESI-Q-TOF/MS) and GC-MS methods to determine the pharmacokinetics and elimination pattern of tramadol in zebrafish tissues and its main phase I metabolites following oral or intramuscular (IM) administration to zebrafish.

\section{Materials and methods}

Drugs and chemicals. Tramadol hydrochloride injection $\left(\mathrm{TH}_{\mathrm{i}}\right)$ was purchased from Medochemie Ltd (Limassol, Cyprus), and tramadol hydrochloride tablets $\left(\mathrm{TH}_{\mathrm{t}}\right)$ were from Wellso
Pharmaceutical Co., Ltd. (Beijing, China). Solvents used were HPLC grade and purchased from Tedia Company, Inc. (Fairfield, OH, USA). Milli-Q water (EMD Millipore, Billerica, MA, USA) was used throughout. Chemicals were all reagent grade.

Animals. Zebrafish (Danio rerio) were obtained from Wei Tong Lihua BioModel Company (Beijing, China) and raised to maturity in a stock tank without filters at a temperature of $28^{\circ} \mathrm{C}$ under cycles of $14 \mathrm{~h}$ light and $10 \mathrm{~h}$ dark. Their daily care conformed a guide for the laboratory use of zebrafish (24).

Experimental fish were randomly selected from the stock tank, and maintained in individual beakers with covers for 2 days. IM administration of $\mathrm{TH}_{\mathrm{i}}$ was performed using a microinjector and self-made equipment with a superficial hole to hold the fish. In this equipment, wet absorbent cotton was fixed to keep the body of the fish wet, above which several rubber bands were used to strengthen the hold. Sites for IM injection were the flanks, usually midpoint between the dorsal fin and the lateral line. A volume of drug or saline was injected into treatment group or control group, respectively, and no treatment was administered to the vehicle group. Fish were placed back into their beakers. For the oral administration of tramadol, zebrafish were pre-exposed for a certain time to a solution of tramadol, with $\mathrm{TH}_{\mathrm{t}}$ directly dissolved into the tank. Experiments were performed in accordance with the guidelines published in the NIH Guide for the Care and Use of Laboratory Animals.

Preparation of samples for ESI-Q-TOF/MS and GC-MS. Zebrafish were randomly divided into 3 groups (n=6 per group). Fish were IM-injected with $4 \mu \mathrm{l}$ saline or $\mathrm{TH}_{\mathrm{i}}(10 \mu \mathrm{g} / \mu \mathrm{l})$, or received no treatment. Tissue samples from brain, eyes, gill, heart, liver, gut and muscle (from the tail of fish, far away from the injection point), were isolated at 0.5 or $1 \mathrm{~h}$ after the treatment, and washed with cold $0.9 \%$ saline four times, dried with tissue paper, weighed and transferred to glass tubes $(1 \mathrm{~cm}$ I.D. on ice). The treatment time for every fish was strictly controlled, from injection to tissue isolation.

Tissue samples with 9-fold (w/v) excess saline were homogenized using a microhomogenizer at 2,000 $\mathrm{rpm} / \mathrm{min}$ for $10 \mathrm{sec}$ and repeated 5 times. A $1 \mathrm{ml}$ aliquot of ethanol was added into the homogenate. The tubes were sealed and shaken in a reciprocal shaker for $2 \mathrm{~h}$, following centrifugation at $12,000 \times \mathrm{g}$ at room temperature for $10 \mathrm{~min}$, and the supernatant was transferred to fresh disposable glass tubes. The ethanol extraction procedure was repeated four times. Pooled the supernatant together, then were evaporated under a stream of nitrogen at $40^{\circ} \mathrm{C}$, finally the residue was stored at $-20^{\circ} \mathrm{C}$ prior to analysis. The residue was dissolved in $\eta$-hexane at the same ratio $(\mathrm{w} / \mathrm{v})$, vortexed and centrifuged at $200 \times \mathrm{g}$ for $10 \mathrm{~min}$ at $4^{\circ} \mathrm{C}$ prior to ESI-Q-TOF/MS and GC-MS analysis.

Pharmacokinetic analysis of tramadol in zebrafish brain tissue using $G C-M S$. The fish were randomly divided into two groups, and received a single IM dose of either 10 or $25 \mu \mathrm{g} / \mu \mathrm{l}$ $\mathrm{TH}_{\mathrm{i}}$. Brains $(\mathrm{n}=7)$ were collected prior to drug administration (pre-injection, time 0 ), and at 1, 3, 5, 15, 25, 35, 45, 75, 120 and $240 \mathrm{~min}$ after a single dose of $10 \mu \mathrm{g}$, or at $1,2,4,5,7,10,20$, 
$30,40,55,95,155,180$ and 300 min after a single injection of $25 \mu \mathrm{g}$. in order to optimize the dose, injection and isolation time. Brain samples were immediately placed on ice, washed with $0.9 \%$ saline ( $\mathrm{pH} 9.0$ ) four times, then the supernatant was discarded following washing with $0.9 \%$ saline and extracted as aforementioned. A $1 \mu l$ aliquot volume of each sample was injected into the GC-MS system. For quantification, calibration curves were created and linearity calculated. A standard calibration curve of tramadol content vs. mean peak area in GC-MS analysis was conducted in the range of $0-10 \mu \mathrm{g}$, by adding $0,0.5,5$, and $10 \mu \mathrm{g}$ tramadol into the brain samples from untreated vehicle control fish and using the aforementioned extraction procedures.

Identification of tramadol primary metabolites in brain tissue. Primary metabolites, M1 and M2 were prepared from tramadol hydrochloride as previously described (25), with certain modifications. Briefly, $218 \mathrm{mg}$ of platinum oxide hydrate was dissolved in $1 \mathrm{ml}$ methanol, and reduced with hydrogen gas for $5 \mathrm{~min}$. Following centrifugation at $200 \mathrm{xg}$ for $10 \mathrm{~min}$ at $4^{\circ} \mathrm{C}$, the precipitated platinum black catalyst was washed twice with $0.5 \mathrm{ml}$ water. Then tramadol hydrochloride (20 mg dissolved in $1 \mathrm{ml}$ of distilled water) was immediately added to the catalyst, and the mixture was stirred for $90 \mathrm{~h}$. The reaction mixture was centrifuged at $200 \mathrm{x} g$ for $10 \mathrm{~min}$ at $4^{\circ} \mathrm{C}$ and the supernatant was removed and the residue was washed with $1 \mathrm{ml}$ water. The combined aqueous phases were treated with $0.5 \mathrm{ml} 3 \mathrm{~mol} / \mathrm{l} \mathrm{NaOH}$ solution and extracted twice with $2 \mathrm{ml}$ of $\eta$-hexane. The hexane phase was evaporated under a stream of nitrogen. The resulting compound was used as the desmethyltramadol reference sample in the GC-MS analysis.

Samples for metabolite analysis were prepared following IM and bath exposure. Fish received a single IM dose of either $\mathrm{TH}_{\mathrm{i}}(25 \mu \mathrm{g} / \mu \mathrm{l})$ or saline, or for bath exposure a solution of $0.06 \mathrm{mg} / \mathrm{ml} \mathrm{TH} \mathrm{t}_{\mathrm{t}}$ was added to the tank for $1 \mathrm{~h}$. Brain samples $(\mathrm{n}=12)$ were obtained at $0.5,1$ and $2 \mathrm{~h}$ after the treatment, which were washed, homogenized and extracted in alkalic conditions as aforementioned, for the reference substance.

ESI-Q-TOF/MS. ESI-Q-TOF mass spectra were recorded on a Bruker Autoflex III TOF/TOF 200 mass spectrometer (Bruker Corporation, Ettlingen, Germany). The parameters were as follows: Ion mode, positive; source temperature, $200^{\circ} \mathrm{C}$; ion spray voltage, $4.5 \mathrm{kV}$; declustering potential, $30 \mathrm{~V}$; and mass range, $75-800 \mathrm{~m} / \mathrm{z}$. A $20 \mu \mathrm{l}$ aliquot of each sample was analyzed by infusion using a syringe pump for direct injection into ion sources, at $2 \mu \mathrm{l} / \mathrm{min}$ flow rate. MS/MS parameters were: Collision energy, $25 \mathrm{~V}$; and collision gas pressure $\left(\mathrm{N}_{2}\right), 4.7 \mathrm{mPa}$.

$G C-M S$. Triple-quadrupol Varian mass spectrometer MS 1200 equipped with a CP-3800 gas chromatograph (Agilent Technologies GmbH, Waldbronn, Germany) was used to detect tramadol and the metabolites. The analytes were separated on a Varian Factor Four VF-5 ms capillary column (30 m x 0.25 mm ID, $0.25 \mu \mathrm{m} \mathrm{df}$; Agilent Technologies $\mathrm{GmbH}$ ) with helium 5.0 as the carrier gas (constant flow, $0.2 \mathrm{ml} / \mathrm{min}$ for tramadol detection and $1 \mathrm{ml} / \mathrm{min}$ for desmethyltramadol detection). The front injector temperature was $260^{\circ} \mathrm{C}$. The oven temperature initially set at $100^{\circ} \mathrm{C}$ for
$1 \mathrm{~min}$, was increased to $220^{\circ} \mathrm{C}$ at $20^{\circ} \mathrm{C} / \mathrm{min}$, held for $10 \mathrm{~min}$, and then increased to $300^{\circ} \mathrm{C}$ at $20^{\circ} \mathrm{C} / \mathrm{min}$, and finally held for 9 min. Injection was performed in splitless mode (10:1; $60 \mathrm{sec}$ delay before opening the splitter). Ionization was performed in electron impact (EI) mode with electron energy of $70 \mathrm{eV}$. Analytes were measured in full scan mode within the mass range, $m / z=50-400$. Selected-ion-monitoring (SIM) mode for quantitative analysis from 6 to $40 \mathrm{~min}$ was used, $\mathrm{m} / \mathrm{z}, 58,263$ for tramadol; $m / z$ 44, 249 for M2; $m / z$ 58, 121, 249 for M1.

\section{Results}

Detection and structural analysis of tramadol in tissues by ESI-Q-TOF/MS. In the current study, two types of tramadol, injection $\left(\mathrm{TH}_{\mathrm{i}}\right)$ and tablets $\left(\mathrm{TH}_{\mathrm{t}}\right)$, with the same main component, were used. The UV absorption spectra of both types were monitored a single well-defined maximum peak either dissolved in $\mathrm{CHCl}_{3}$ or water at $271 \mathrm{~nm}$, in the wavelength range of 200-400 $\mathrm{nm}$ with a fixed slit width of $5 \mathrm{~nm}$ (Fig. 1A). Furthermore, only a single molecular ion peak was detected by ESI-Q-TOF/MS or GC-MS, at $\mathrm{m} / \mathrm{z} 264.2578$ (Fig. 1B) and 263 (Fig. 1C), respectively. No structural differences were observed, and no interfering absorbance was detected. Therefore, both types were used as the parallel treatment groups in the subsequent experiments.

Total mass spectra of the brain tissue samples of zebrafish are presented in Fig. 2A. By comparing the tramadol group with the untreated group, an ion peak at $m / z 264$ in every tissue from tramadol administration group was easily detected (data not shown). Chosen parent peaks for further ionization, seven high-resolution fragment ion peaks were identified, namely at $\mathrm{m} / \mathrm{z}$ 58.3564, 101.3825, 129.7751, 171.2543, 200.4007, 222.2337 and 264.4308 (Fig. 2B), and the molecular weight differences between the two adjacent fragments were 43, 28, 42, 29, 22 and 42, respectively. Among them, the peak at $\mathrm{m} / \mathrm{z} 58.3564$ corresponded to $\left[\mathrm{C}_{3} \mathrm{H}_{8} \mathrm{~N}\right]$, and on this basis, new chemical structures were added one by one according to the differences, and a series of structures were established, $\left[\mathrm{C}_{6} \mathrm{H}_{15} \mathrm{~N}\right]$, $\left[\mathrm{C}_{8} \mathrm{H}_{19} \mathrm{~N}\right],\left[\mathrm{C}_{10} \mathrm{H}_{21} \mathrm{NO}\right],\left[\mathrm{C}_{12} \mathrm{H}_{25} \mathrm{NO}\right],\left[\mathrm{C}_{14} \mathrm{H}_{25} \mathrm{NO}\right]$, and finally the structure of tramadol $\left[\mathrm{C}_{16} \mathrm{H}_{25} \mathrm{NO}_{2}\right]$ was obtained. The details of the whole process of structure analysis are presented in Table I. Thus, ESI-Q-TOF/MS is considered to be a rapid and adequately sensitive method for the detection of tramadol distribution in tissues.

Tramadol distribution in zebrafish tissues. The compounds of the brain extract in the hexane fraction, with or without tramadol treatment, were performed to optimize the analysis conditions. Comparing the chemical compositions of brain extract from control with tramadol IM administration, the peaks at $m / z 263$ were only detected in the tramadol group, without any interfering peak from other compounds, within retention time of $13.824 \mathrm{~min}$. It was exactly the tramadol peak, identified by comparison of its mass spectra with that from the software library, which was further confirmed using SIM mode by monitoring for tramadol and $\left[\mathrm{C}_{3} \mathrm{H}_{8} \mathrm{~N}\right]^{+}$at $\mathrm{m} / z 263$ and 58 , respectively.

Total ion chromatograms of extracts from brain tissues, and enlarged portion of ion chromatograms from other six other tissues are presented in Fig. 3. Using full scan 
Table I. $m / z$, molecular formula and chemical structure of fragment ions from tramadol.

\begin{tabular}{|c|c|c|}
\hline $\mathrm{m} / \mathrm{z}$ & Formula molecular & Chemical structure \\
\hline \multicolumn{3}{|c|}{ ESI-Q-TOF/MS } \\
\hline 58.3564 & {$\left[\mathrm{C}_{3} \mathrm{H}_{8} \mathrm{~N}\right]$} & \\
\hline 101.3825 & {$\left[\mathrm{C}_{6} \mathrm{H}_{15} \mathrm{~N}\right]$} & \\
\hline 129.7751 & {$\left[\mathrm{C}_{8} \mathrm{H}_{19} \mathrm{~N}\right]$} & \\
\hline 171.2543 & {$\left[\mathrm{C}_{10} \mathrm{H}_{21} \mathrm{NO}\right]$} & \\
\hline 200.4007 & {$\left[\mathrm{C}_{12} \mathrm{H}_{25} \mathrm{NO}\right]$} & \\
\hline 222.2337 & {$\left[\mathrm{C}_{14} \mathrm{H}_{25} \mathrm{NO}\right]$} & \\
\hline 264.4308 & {$\left[\mathrm{C}_{16} \mathrm{H}_{25} \mathrm{NO}_{2}\right]$} & \\
\hline
\end{tabular}

GC-MS

188

$$
\left[\mathrm{C}_{13} \mathrm{H}_{16} \mathrm{O}\right]
$$

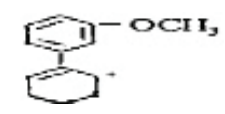

218

$$
\left[\mathrm{C}_{15} \mathrm{H}_{24} \mathrm{~N}\right]
$$

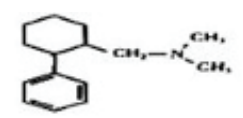

ESI-Q-TOF/MS, electrospray ionization-quadrupole-time of flight/mass spectrometry; GC-MS, gas chromatography/mass spectrometry.

or SIM mode, tramadol peaks were detected in all tissues from the tramadol administration group, at 0.5 or $1 \mathrm{~h}$ after injection. The results were highly consistent with that of ESI-Q-TOF/MS. For each tissue, the sample was weighed, prepared by the same process, and dissolved in $\eta$-hexane at the same ratio $(\mathrm{w} / \mathrm{v})$. Therefore, the same tissue type from different treatment times can be compared. The mean peak area obtained by three repeated detections was substituted into the formula, namely the standard calibration curve of tramadol content vs. the mean peak area: $\mathrm{y}=0.3669 \mathrm{x}-0.0184$ $\left(\mathrm{R}^{2}=0.999,0 \leq \mathrm{x} \leq 10 \mu \mathrm{g}\right)$ or $\mathrm{y}=24.883 \mathrm{x}-95.96\left(\mathrm{R}^{2}=0.965\right.$, $50 \leq \mathrm{x} \leq 450 \mu \mathrm{g})$. Quantification of tramadol in tissues was compared, at 0.5 or $1 \mathrm{~h}$ after injection. Quantifying the trend of tramadol in different tissue was heterogeneous over time. Compared with its quantification at $0.5 \mathrm{~h}$, tramadol in liver and gut tissues, was observably increased as much as 56.4 and 742.2 times in the following $0.5 \mathrm{~h}$, respectively. In eyes, muscle and gill tissues, tramadol was maintained at relatively stable low levels, with only 1.45 -fold increase, and 0.88 or 0.92 -fold decrease comparing between $0.5 \mathrm{~h}$ and $1 \mathrm{~h}$, respectively. However, brain and heart tissue experienced a significant decrease, with 0.096 and 0.01 fold decrease at $1 \mathrm{~h}$ compared with $0.5 \mathrm{~h}$ (Fig. 3).
Pharmacokinetics of tramadol in zebrafish brain. The tramadol content in the brain after IM administration was presented in Fig. 4. The standard calibration curve in Fig. 4A (indicated by arrow; $\mathrm{y}=0.3669 \mathrm{x}-0.0184 ; \mathrm{R}^{2}=0.999$, linear range from 0 to $10 \mu \mathrm{g}$ ), demonstrated a highly linear relationship between the concentration and its peak area in the GC spectra. A 3-exponential model best described the brain concentrations of tramadol for the 10 and $25 \mu \mathrm{g}$ treatments $(n=7)$. With different doses of IM tramadol administration and detection time, the concentrations in the brain were similar for injection of 10 (Fig. 4A) and $25 \mu \mathrm{g}$ (Fig. 4B). The curve indicated an initial rapid phase, corresponding to the detection of the tramadol within $1 \mathrm{~min}$, and reached peak value at 5 min $\left(\mathrm{T}_{\max }\right)$, after a single dose of 25 and $10 \mu \mathrm{g}$ IM drug injection. The peak contents of tramadol were $1.76\left(\mathrm{C}_{\max }\right)$ and $0.68 \mu \mathrm{g}\left(\mathrm{C}_{\text {max }}\right)$ for the 25 and $10 \mu \mathrm{g}$ injections, respectively, and the ratio between two values was quite similar to that of initial dosage. Despite similar $\mathrm{T}_{\max }$, faster drug clearance was observed with the low-dose group, with the content dropped to initial value $(1.11 \mu \mathrm{g})$ by $20 \mathrm{~min}$ and was detectable up to $3 \mathrm{~h}$ in the low-dose group, however, it took $80 \mathrm{~min}$ to fall to initial value $(1.76 \mu \mathrm{g})$ in high-dose groups, and tramadol was detectable up to $4 \mathrm{~h}$ after the treatment.

Detection of two primary metabolites, $M 1$ and $M 2$, in brain tissue. Primary desmethyltramadol reference substances produced using the previously reported method (25), and were analyzed by GC-MS. Compared with the aforementioned strategies to detect tramadol alone, the extraction method and analysis conditions were optimized for combined determination of tramadol and its primary metabolites. The total ion chromatogram (Fig. 5A) and related EI mass spectrograms were obtained (Fig. 5B-D). As presented in Fig. 5A, the three compounds (tramadol, M2 and M1) were separately detected at 9.096, 9.417 and $9.573 \mathrm{~min}$. Data searching in the library, their EI mass spectra consistent with those of tramadol, M2 and M1.

Brain extractions from the IM and bath exposure treatment groups at $0.5,1$ and $2 \mathrm{~h}$ were analyzed under the same conditions, and magnified chromatograms are presented in Fig. 6. Three novel peaks were present in all the tramadol treatment groups compared with the control group, even with different treatment strategies, at different sampling times, and using different concentrations. The elution time and EI mass spectra with were consistent with those of the tramadol, M2 and M1 reference samples. Furthermore, distinct changes in tramadol, M1 and M2 concentrations were observed following the two different types of administrations. For example, following IM administration, the concentration of tramadol was very high and gradually decreased between 0.5 and $2 \mathrm{~h}$. A higher concentration of $\mathrm{M} 2$ was detected at $1 \mathrm{~h}$ compared with 0.5 and $2 \mathrm{~h}$ IM treatments. However, in the bath exposure treatment groups, the concentration of tramadol was higher at $1 \mathrm{~h}$ than at 0.5 and $2 \mathrm{~h}$. The concentration of M2 was variable, with higher values observed at 0.5 and $2 \mathrm{~h}$, and lower at $1 \mathrm{~h}$. Unfortunately, for M1, data analysis was not always possible due to a very low and variable concentration. Thus, two primary metabolites M1 and M2 were detected, however the total amount of M2 was greater than M1, as the main desmethyl metabolite detected in zebrafish brain. 
A

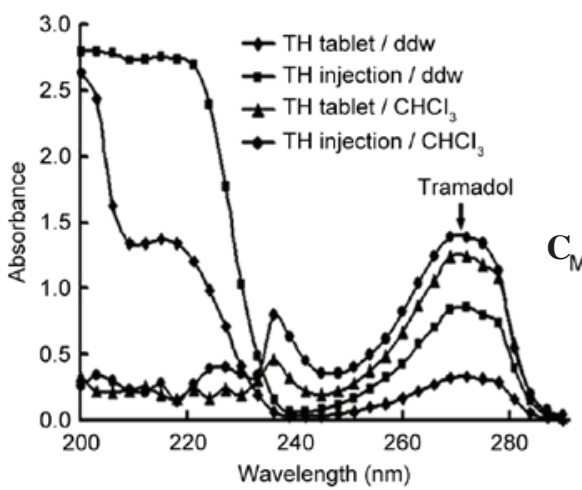

B

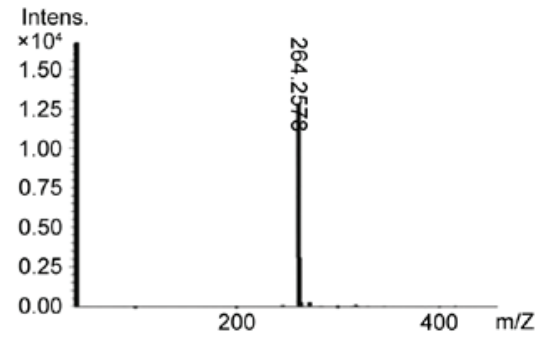

$\mathrm{C}_{\mathrm{M} \text { cou }}$

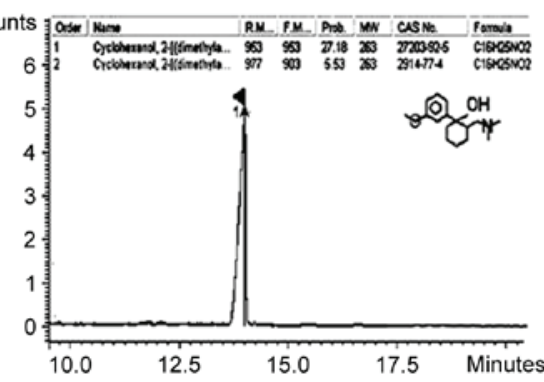

Figure 1. Characteristics of TH. (A) UV spectra of TH; (B) molecular weight analysis tramadol hydrochloride by electrospray ionization-quadrupole-time of flight/mass spectrometry analysis. (C) Analysis and identification of tramadol by gas chromatography/mass spectrometry. TH, tramadol hydrochloride; $\mathrm{ddw}$, double distilled water.

A
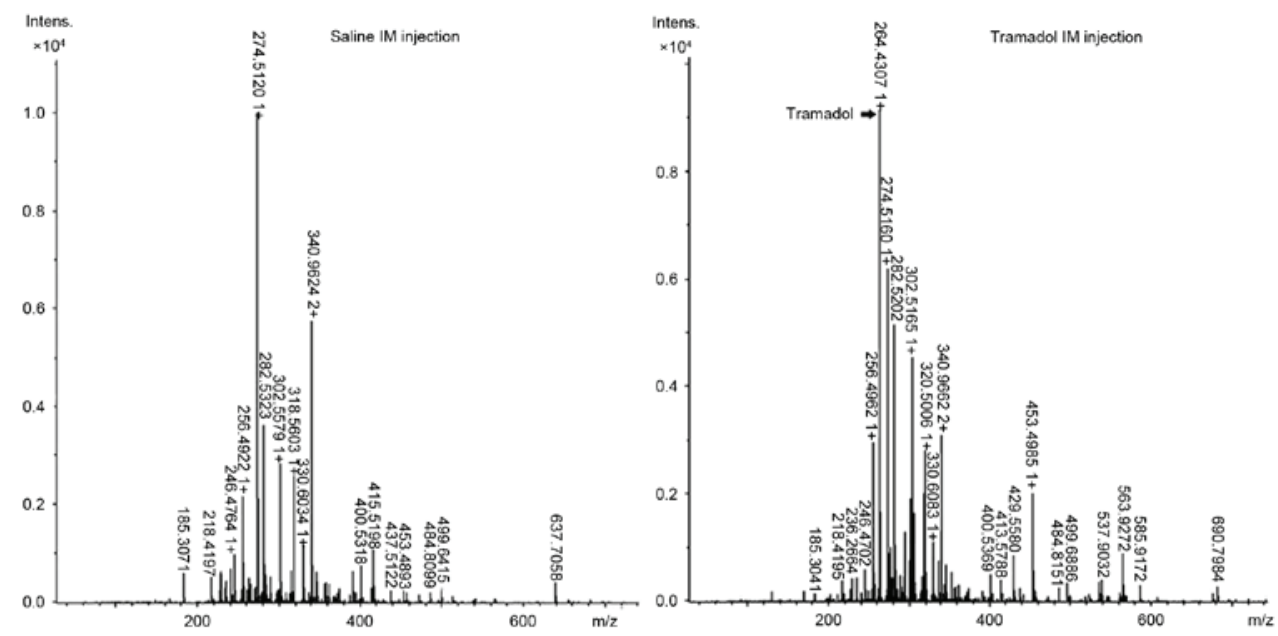

B

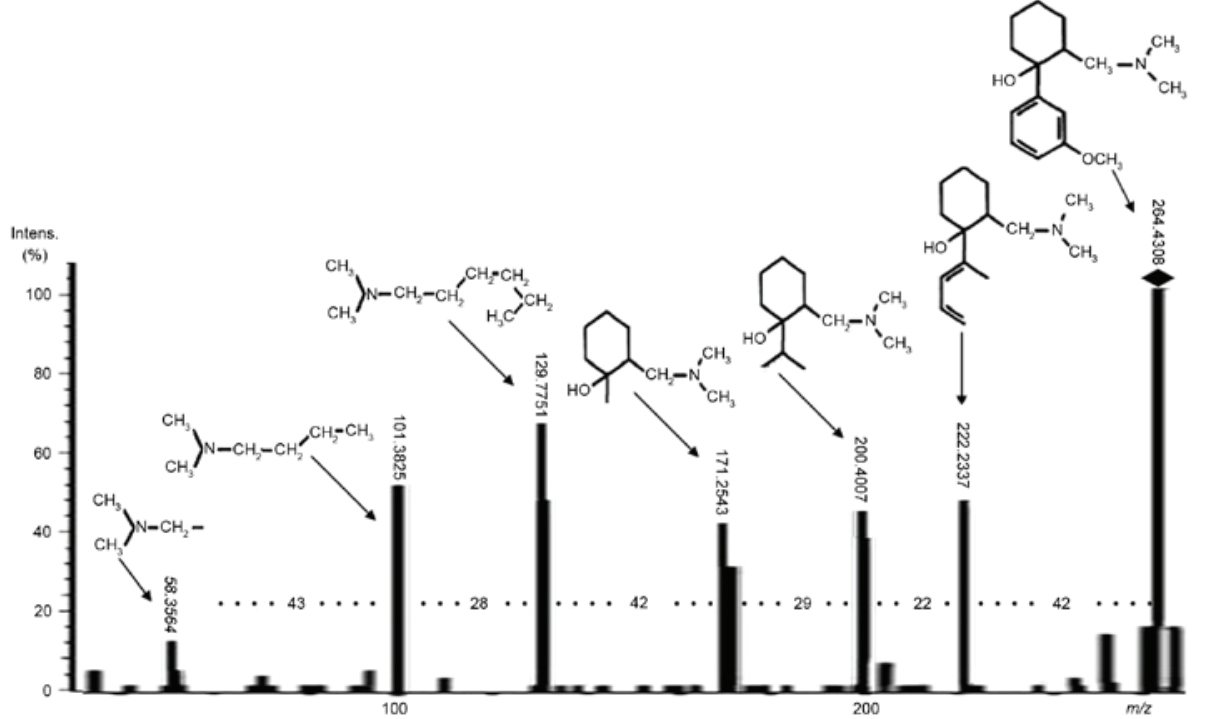

Figure 2. Total mass spectrograms of extractions from brain tissues by (A) electrospray ionization quadrupole time of flight/mass spectrometry analysis (left, saline IM injection administration; right, tramadol hydrocholride IM injection administration) and (B) by gas chromatography/mass spectrometry. IM, intramuscular. 

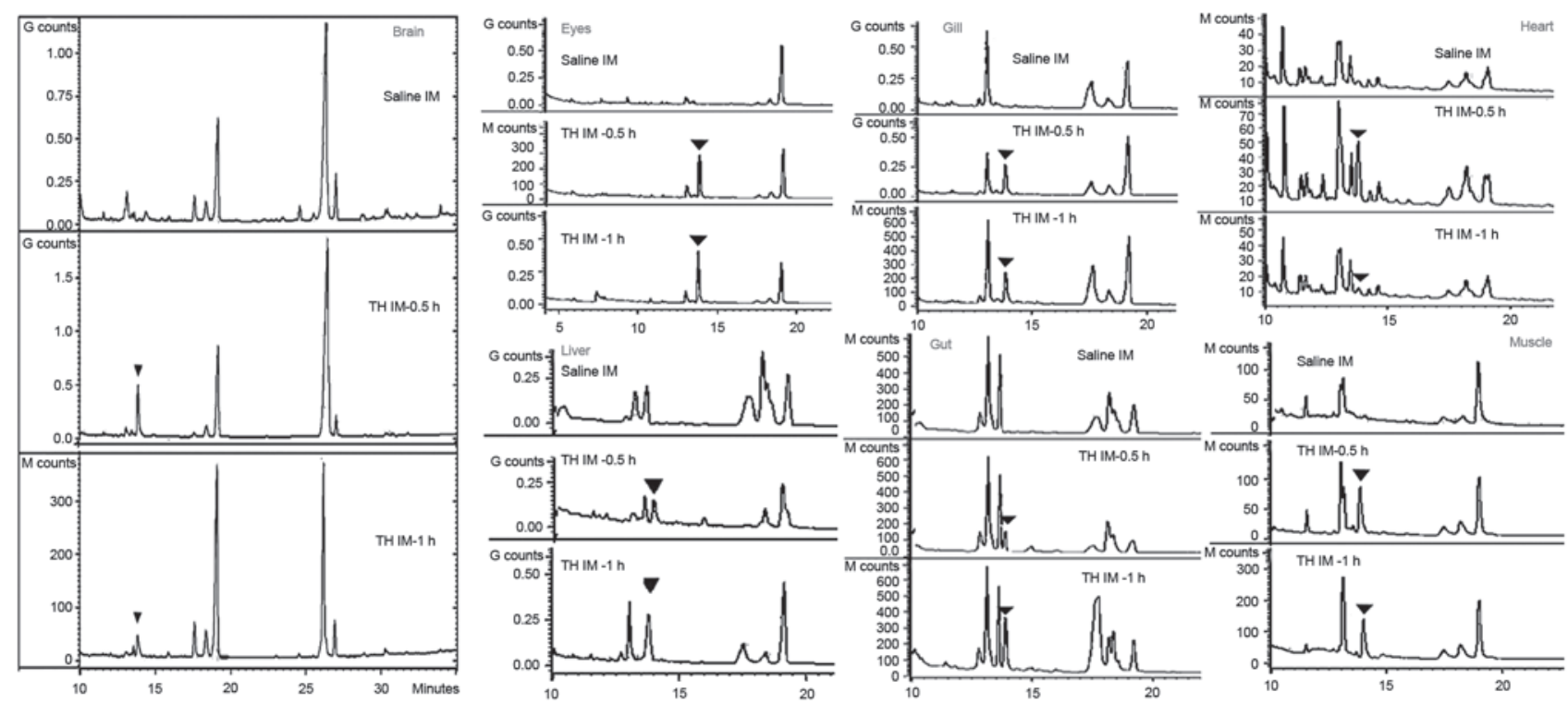

Figure 3. Total ion chromatograms of extractions from tissues (brain, eyes, gill, heart, liver, gut, muscle) and the data was obtained by electrospray ionization-quadrupole-time of flight/mass spectrometry. M, mega; G, giga.

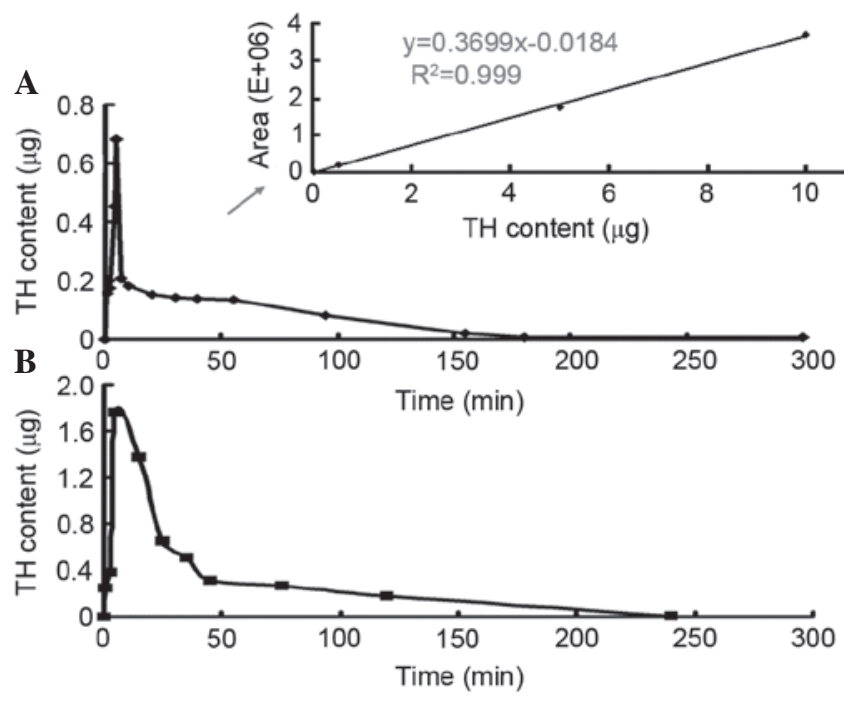

Figure 4. Time-course of tramadol content in brain tissue of zebrafish administered a single intramuscular dose of (A) 10 and (B) $25 \mu \mathrm{g}$ tramadol, and standard calibration curve of mean peak area vs. tramadol content (indicated by arrow), and the data was obtained by gas chromatography/mass spectrometry. TH, tramadol hydrochloride.

\section{Discussion}

Tramadol is a centrally acting analgesic with opiate, adrenergic and serotoninergic interactions. The current study examined the distribution and elimination of tramadol in zebrafish tissues, the pharmacokinetic profiles and the two major metabolites of tramadol, M2 and M1, in brain tissue. This was performed using ESI-Q-TOF/MS and GC-MS, to the best of our knowledge this is the first report of generally applicable methods for the determination of tramadol concentration in various tissues, showed that tramadol distribution and residence in tissues was rapid separated from other compounds, and further structure identified by mass spectrometry. However, due to signal accumulation used to improve the signal-to-noise ratio and the abundant cluster of ion signals, it remained difficult to quantify and compare the tramadol concentrations, and to detect the trace amounts of metabolites by ESI-Q-TOF/MS.

Due to its high resolution, GC-MS has solved the issues with quantification and identification of minor components within a mixture. The drug distribution in the present study demonstrated that, following IM administration, tramadol was absorbed and distributed to other tissues via the circulatory system. The drug was eliminated very slowly in the eyes, muscle and gills, which may maintain the effects of low drug dosage in these tissues for a long time; however in heart and brain tissue the tramadol and the metabolites were rapidly eliminated. Finally, in the liver and gut (tissues involved in drug metabolism and elimination) the drug concentration was increased over time.

No adverse effects were observed following tramadol administration in several animal species in previous studies, however in horses, nausea, confusion, agitation, tremors and tachycardia were reported $(26,27)$. In the current study, behavior observations demonstrated that an increased preference for the oxygen-rich surface water layer in fish, accompanied by a significant reduction in activity, following tramadol IM administration or bath exposure. After tramadol IM administration, fish would gather together at the surface of the water within $1 \mathrm{~min}$. When the given dosage was increased to $50 \mu \mathrm{g}$, the majority of fish moved to the bottom of the tank and were dead within 4-5 min. However, a small number of fish slowly recovered and swam to the water surface layer after $\sim 20 \mathrm{~min}$, whereas, this abnormal behavior was not observed when the fish were exposed to pethidine hydrochloride (a more effective analgesic) or in the saline control group. The time-course of abnormal behavior following treatment with tramadol was highly similar to the pharmacokinetic distribution and elimination trends of tramadol in zebrafish tissues.

The pharmacokinetic profiles and metabolism of tramadol have been previously reported in humans, mice, camels, 
A

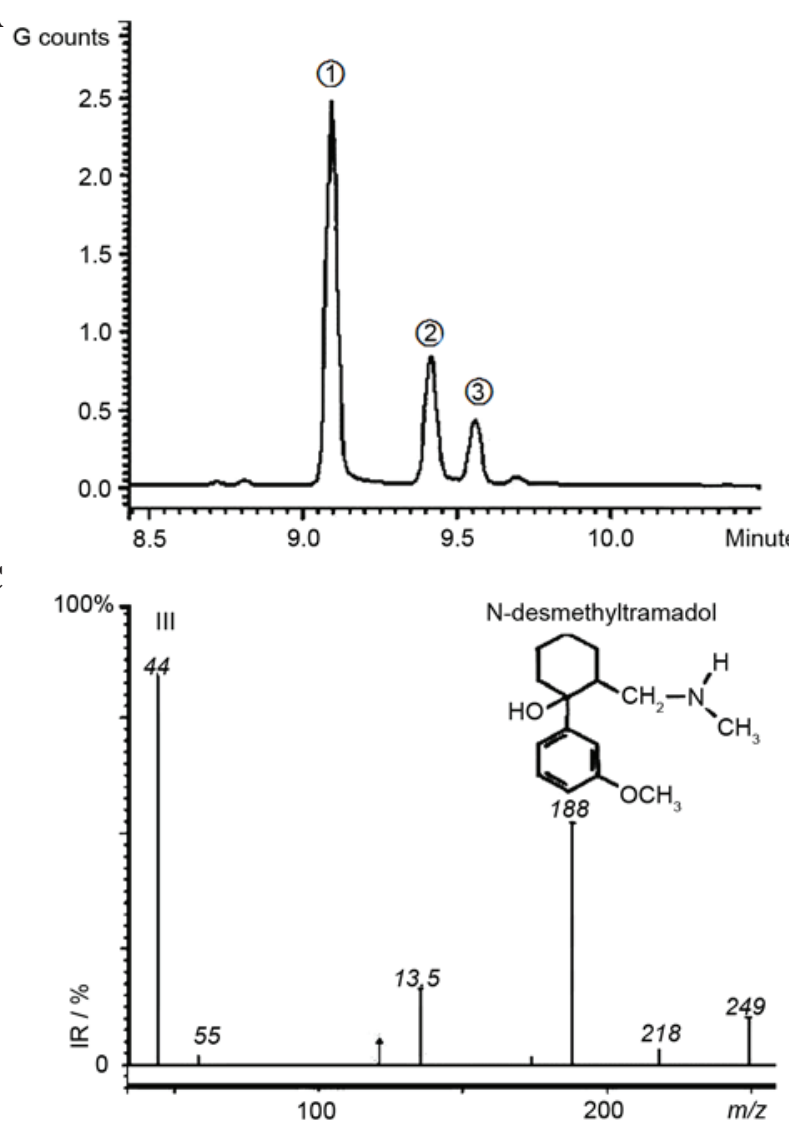

B

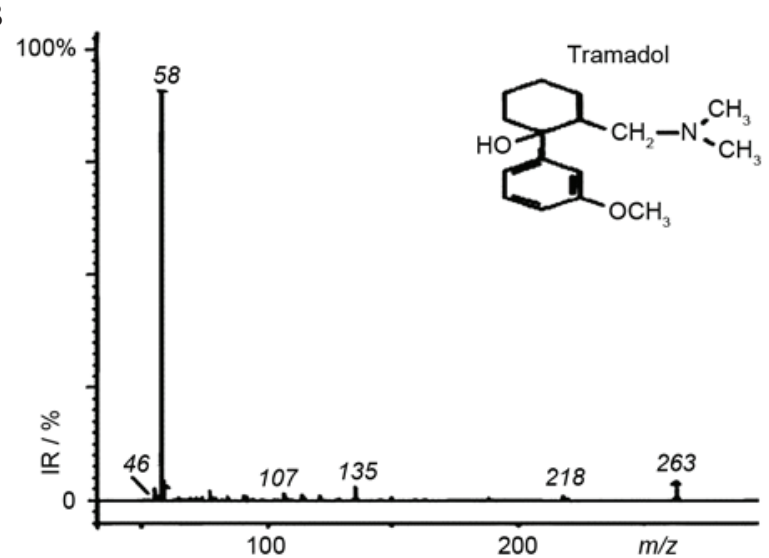

D

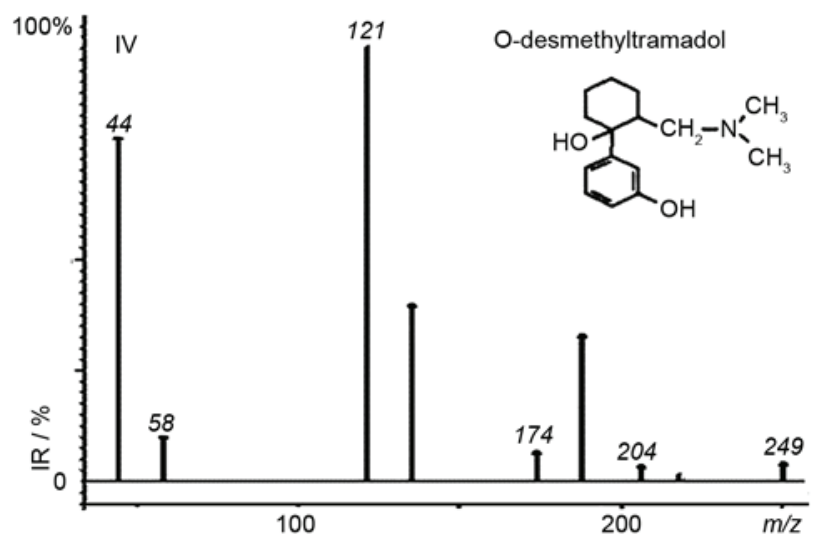

Figure 5. (A) Total ion chromatogram. Peak (1), tramadol; peak (2), N-desmethyltramadol; peak (3), O-desmethyltramadol. Electron impact mass spectrograms of (B) tramadol, (C) N-and (D) O-desmethyltramadol. The data was obtained by gas chromatography/mass spectrometry.

A

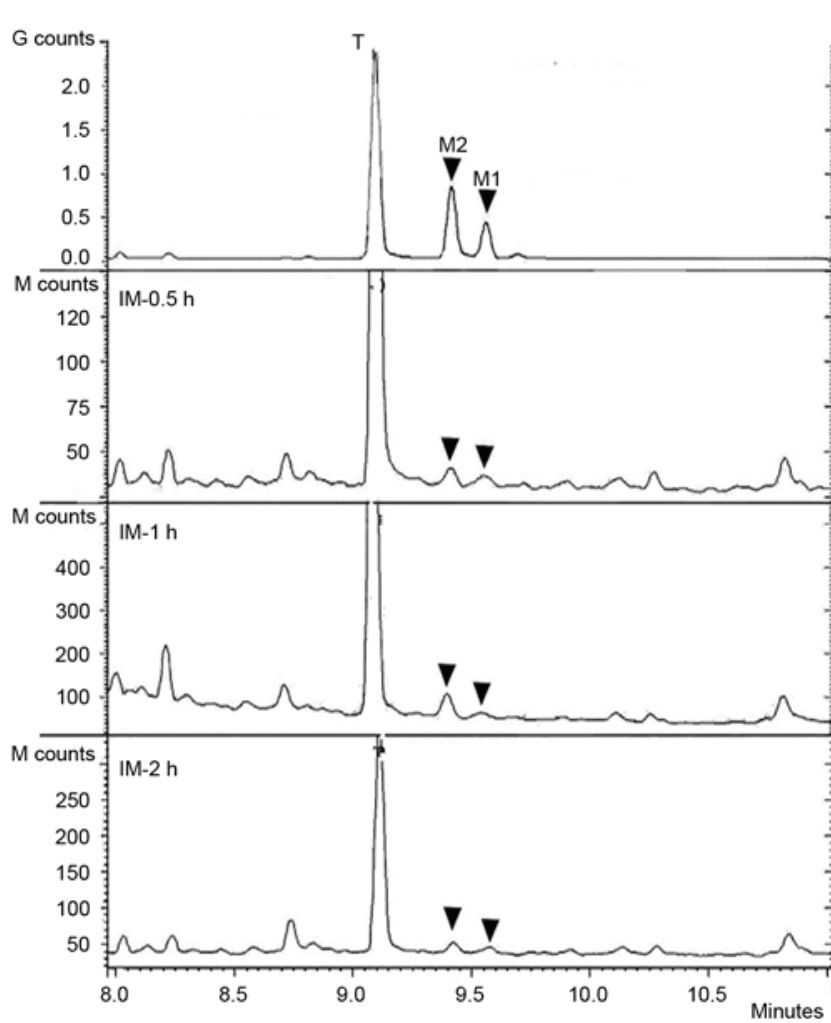

B

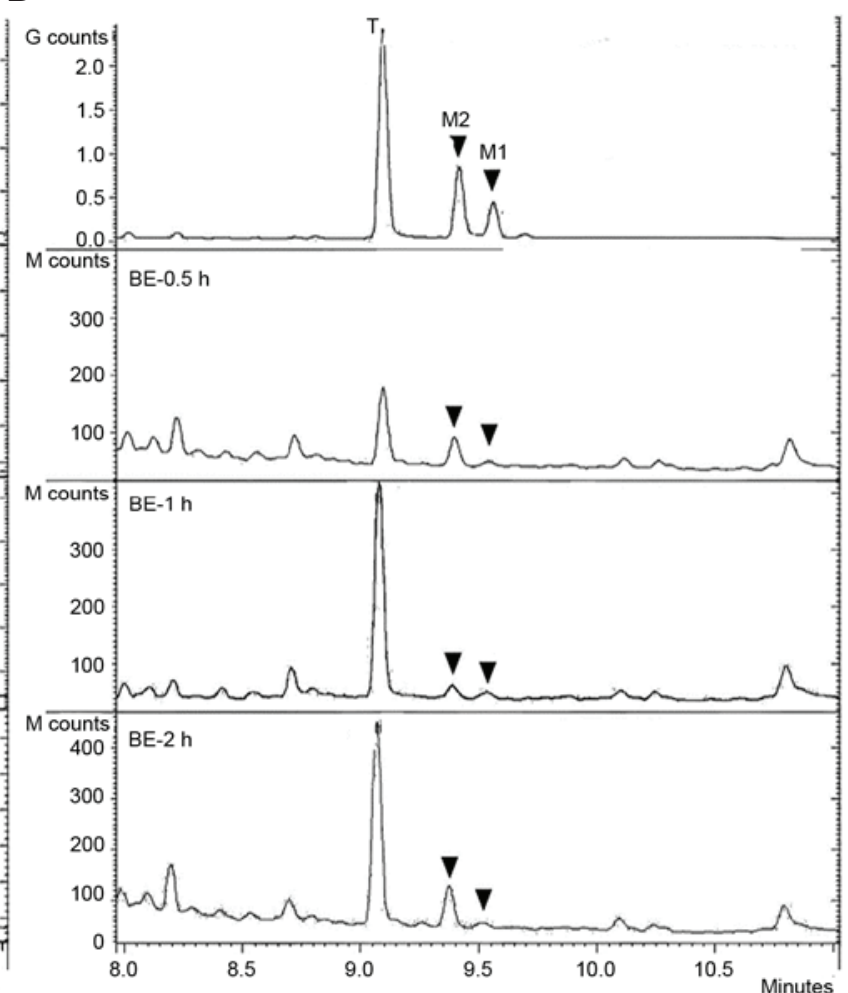

Figure 6. Ion chromatogram of tramadol primary metabolites in brain tissue after (A) IM or (B) BE administration (IM-0.5, 1, 2 h or BE-0.5, 1, $2 \mathrm{~h}$ after tramadol hydrochloride IM or BE administration). The data was obtained by gas chromatography/mass spectrometry. IM, intramuscular; BE, bath exposure; T tramadol; M2, N-desmethyltramadol; M1, O-desmethyltramadol. 
hamsters, rats, guinea-pigs, rabbits and dogs, however, to the best of our knowledge, this is the first study describing the only kinetic parameters of tramadol and its $\mathrm{N}$ - and $\mathrm{O}$-metabolites in zebrafish brain tissue. The primary phase I metabolites, namely, M1 and M2, and similar metabolites are produced but in different amounts. In humans, M1 has been reported to be the major metabolite, however previous studies demonstrated that M1 was only marginally produced in equine species, with M2 the major metabolite produced (22). In the present study, M2 concentrations in the tissues were higher than M1, indicating that M2 was the major metabolite produced in brain tissue, by both routes of administration (IM and BE). Although relatively low concentrations of M1 were obtained in brain tissue, the clinical response to tramadol is closely correlated with its metabolism, because of the different analgesic activity of its metabolites. M1 is reported to be the main active metabolite that determines the effectiveness of the drug, because it has an affinity on $\mu$-opioid receptor 200 -fold higher than that of the parent drug. Another metabolite with a higher affinity than tramadol for the $\mu$-opioid receptor is M5, however in a previous study M5 was not detected in brain tissue, and has been demonstrated to not penetrate the blood-brain barrier (BBB) because of its high polarity (28). Zebrafish emerged as a useful model organism to investigate the effect on drugs on the brain, as its BBB is similar to that of higher vertebrates (29). Therefore, in vivo M1 appears to be responsible for the $\mu$-opioid-derived analgesic effect of tramadol, and zebrafish are likely to be an appropriate experimental model to analyze the biological effects of drug abuse and the neurotoxicity of tramadol.

In conclusion, the present study developed a simple, cost effective, robust and high throughput analytical method for simultaneous estimation of the levels of tramadol and its primary metabolites in zebrafish tissues. The pharmacokinetics and metabolism data reported in the current study provides information for the design of future studies involving the use of zebrafish as a model organism for investigating drug mechanism and neurotoxicity, and for additional potential screening of tramadol.

\section{Acknowledgements}

This work was financially supported by grants from the National Natural Scientific Foundation of China (grant nos. 81000769 and 81370048 ).

\section{References}

1. Grond S and Sablotzki A: Clinical pharmacology of tramadol. Clin Pharmacokinet 43: 879-923, 2004

2. Ebrahimzadeh H, Mollazadeh N, Asgharinezhad AA, Shekari N and Mirbabaei F: Multivariate optimization of surfactant-assisted directly suspended droplet microextraction combined with GC for the preconcentration and determination of tramadol in biological samples. J Sep Sci 36: 3783-3790, 2013.

3. El-Sayed AA, Mohamed KM, Nasser AY, Button J and Holt DW: Simultaneous determination of tramadol, O-desmethyltramadol and $\mathrm{N}$-desmethyltramadol in human urine by gas chromatography-mass spectrometry. J Chromatogr B Analyt Technol Biomed Life Sci 926 : 9-15, 2013.

4. Ardakani YH, Mehvar R, Foroumadi A and Rouini MR: Enantioselective determination of tramadol and its main phase I metabolites in human plasma by high-performance liquid chromatography. J Chromatogr B Analyt Technol Biomed Life Sci 864: 109-115, 2008.
5. Epstein DH, Preston KL and Jasinski DR: Abuse liability, behavioral pharmacology and physical-dependence potential of opioids in humans and laboratory animals: Lessons from tramadol. Biol Psychol 73: 90-99, 2006.

6. Ferrari A, Tiraferri I, Palazzoli F and Licata M: Tramadol abuse in a binge pattern in a young depressed woman. Eur Addict Res 20: 82-86, 2014.

7. Ozok HU, Sagnak L, Ates MA, Karakoyunlu N, Topaloglu H and Ersoy H: The efficiency of a sedative or analgesic supplement to periprostatic nerve blockage for pain control during transrectal ultrasound-guided prostate biopsy-a prospective, randomized, controlled, double blind study. Arch Med Sci 6: 787-792, 2010

8. Sanchez-Simon FM and Rodriguez RE: Developmental expression and distribution of opioid receptors in zebrafish. Neuroscience 151: 129-137, 2008.

9. Dooley K and Zon LI: Zebrafish: A model system for the study of human disease. Curr Opin Genet Dev 10: 252-256, 2000.

10. Schenk S and Partridge B: Sensitization and tolerance in psychostimulant self-administration. Pharmacol Biochem Behav 57: 543-550, 1997.

11. Lockwood B, Bjerke S, Kobayashi K and Guo S: Acute effects of alcohol on larval zebrafish: A genetic system for large-scale screening. Pharmacol Biochem Behav 77: 647-654, 2004.

12. Zhu W, Li Y, Kream RM and Stefano GB: Chronic alcohol exposure increases ganglia endogenous morphine levels. Arch Med Sci 6: 316-320, 2010.

13. Gonzalez-Nuñez V, Marrón Fernández de Velasco E, Arsequell G, Valencia G and Rodríguez RE: Identification of dynorphin a from zebrafish: A comparative study with mammalian dynorphin A. Neuroscience 144: 675-684, 2007.

14. Sha YF, Shen S and Duan GL: Rapid determination of tramadol in human plasma by headspace solid-phase microextraction and capillary gas chromatography-mass spectrometry. J Pharm Biomed Anal 37: 143-147, 2005.

15. Musshoff F, Madea B, Stuber F and Stamer UM: Enantiomeric determination of tramadol and O-desmethyltramadol by liquid chromatography- mass spectrometry and application to postoperative patients receiving tramadol. J Anal Toxicol 30: 463-467, 2006.

16. Curticapean A, Muntean D, Curticapean M, Dogaru M and Vari C: Optimized HPLC method for tramadol and O-desmethyl tramadol determination in human plasma. J Biochem Biophys Methods 70: 1304-1312, 2008.

17. Tao Q, Stone DJ Jr, Borenstein MR, Jean-Bart V, Codd EE, Coogan TP, Desai-Krieger D, Liao S and Raffa RB: Gas chromatographic method using nitrogen-phosphorus detection for the measurement of tramadol and its O-desmethyl metabolite in plasma and brain tissue of mice and rats. J Chromatogr B Biomed Sci Appl 763: 165-171, 2001.

18. Tao Q, Stone DJ, Borenstein MR, Codd EE, Coogan TP, Desai-Krieger D, Liao S and Raffa RB: Differential tramadol and O-desmethyl metabolite levels in brain vs. plasma of mice and rats administered tramadol hydrochloride orally. J Clin Pharm Ther 27: 99-106, 2002.

19. Elghazali M, Barezaik IM, Abdel Hadi AA, Eltayeb FM, Al Masri J and Wasfi IA: The pharmacokinetics, metabolism and urinary detection time of tramadol in camels. Vet J 178: 272-277, 2008.

20. Giorgi M, Del Carlo S, Saccomanni G, ŁebkowskaWieruszewska B and Kowalski CJ: Pharmacokinetics of tramadol and its major metabolites following rectal and intravenous administration in dogs. N Z Vet J 57: 146-152, 2009.

21. Vettorato E, Zonca A, Isola M, Villa R, Gallo M, Ravasio G, Beccaglia M, Montesissa $\mathrm{C}$ and Cagnardi P: Pharmacokinetics and efficacy of intravenous and extradural tramadol in dogs. Vet J 183: 310-315, 2010.

22. Saccomanni G, Del Carlo S, Giorgi M, Manera C, Saba A and Macchia M: Determination of tramadol and metabolites by HPLC-FL and HPLC-MS/MS in urine of dogs. J Pharm Biomed Anal 53: 194-199, 2010.

23. Brondani JT, Luna SP, Marcello GC and Padovani CR: Perioperative administration of vedaprofen, tramadol or their combination does not interfere with platelet aggregation, bleeding time and biochemical variables in cats. J Feline Med Surg 11: 503-509, 2009.

24. Westerfield M: The Zebrafish Book. A guide for the laboratory use of zebrafish (Danio rerio). 4th edition. University of Oregon Press, Eugene, OR, 2007. 
25. Leis HJ, Fauler G and Windischhofer W: Synthesis of d1-N-ethyltramadol as an internal standard for the quantitative determination of tramadol in human plasma by gas chromatography-mass spectrometry. J Chromatogr B Analyt Technol Biomed Life Sci 804 369-374, 2004.

26. Ardakani YH and Rouini MR: Pharmacokinetics of tramadol and its three main metabolites in healthy male and female volunteers. Biopharm Drug Dispos 28: 527-534, 2007.

27. Shilo Y, Britzi M, Eytan B, Lifschitz T, Soback S and Steinman A: Pharmacokinetics of tramadol in horses after intravenous, intramuscular and oral administration. J Vet Pharmacol Ther 31: 60-65, 2008.
28. Gillen C, Haurand M, Kobelt DJ and Wnendt S: Affinity, potency and efficacy of tramadol and its metabolites at the cloned human mu-opioid receptor. Naunyn Schmiedebergs Arch Pharmacol 362: 116-121, 2000.

29. Jeong JY, Kwon HB, Ahn JC, Kang D, Kwon SH, Park JA and Kim KW: Functional and developmental analysis of the blood-brain barrier in zebrafish. Brain Res Bull 75: 619-628, 2008 . 